\title{
Effectual Routing in Wireless Sensor Networks- a Review
}

\author{
Gurpreet Singh Chhabra ${ }^{1}$, Dr. P. S. Patheja ${ }^{2}$ \\ HOD (CS), PhD Scholar ${ }^{1}$ \\ Associate Professor, Dean (M.Tech) ${ }^{2}$
}

\begin{abstract}
A wireless sensor network is an enormous assortment of sensor nodes with inadequate power supply and self-conscious computational competence. Due to the circumscribed communication range and high density of sensor nodes, packet dispatching in sensor networks is usually performed through multi-hop data transmission. For that reason, routing in wireless sensor networks has been deliberated as an imperative field of research over the past decade. These days, multipath routing techniques is widely used in wireless sensor networks to improve network performance over and done with efficient utilization of accessible network resources. Consequently, the main intention of this review is to present the conception of the various routing approaches and its fundamental experiments, as well as the basic motivations for exploiting these techniques in wireless sensor networks. Routing procedures for WSNs are responsible for selecting and maintaining the routes in the network and ensure reliable and effective communication in inadequate periods. The energy constriction of WSNs make energy saving become the most significant objective of various routing algorithms. In this paper, a review of routing protocols and algorithms used in WSNs is presented with energy efficiency as the main goalmouth.
\end{abstract}

Keywords: Wireless Sensor Networks, Routing Protocols, energy efficiency, WSN routing protocol, Location aware, scalability, hierarchical, Clustering, Review.

\section{INTRODUCTION}

Wireless sensor networks have critical applications in sensor networks [3]. Meanwhile, designing suitable the scientific, medical, commercial, and military routing protocols to fulfill different performance demands domains. Examples of these applications include of various applications is considered as an important issue environmental monitoring, smart homes and offices, in wireless sensor networking. In this context, researchers surveillance, and intelligent transportation systems. It also have proposed numerous routing protocols to improve has significant usages in biomedical field. As social performance demands of different applications through the reliance on wireless sensor network technology increases, network layer of wireless sensor networks protocol stack we can expect the size and complexity of individual [4,5].

networks as well as the number of networks to increase dramatically.

Wireless sensor networks are typically used in highly dynamic, and hostile environments with no human existence (unlike conventional data networks), and therefore, they must be tolerant to the failure and loss of connectivity of individual nodes. The sensor nodes should be intelligent to recover from failures with minimum human involvement. Networks should support process of autonomous formation of connectivity, addressing, and routing structures.

Recent advances in wireless communication technologies and the manufacture of inexpensive wireless devices have led to the introduction of low-power wireless sensor networks. Due to their ease of deployment and the multifunctionality of the sensor nodes, wireless sensor networks have been utilized for a variety of applications such as healthcare, target tracking, and environment monitoring [1]. The main responsibility of the sensor nodes in each application is to sense the target area and transmit their collected information to the sink node for further operations. Resource limitations of the sensor nodes and unreliability of low-power wireless links [2], in combination with various performance demands of different applications impose many challenges in designing efficient communication protocols for wireless

\section{WIRELESS SENSOR NETWORK CHARACTERISTICS}

A wireless sensor network (WSN) is a network that is made of hundreds or thousands of sensor nodes which are densely deployed in an unattended environment with the capabilities of sensing, wireless communications and computations (i.e. collecting and disseminating environmental data). These spatially distributed autonomous devices cooperatively monitor physical and environmental conditions, such as temperature, sound, vibration, pressure, motion or pollutants, at different locations. The basic architecture of Wireless sensor Network is shown in Figure1.

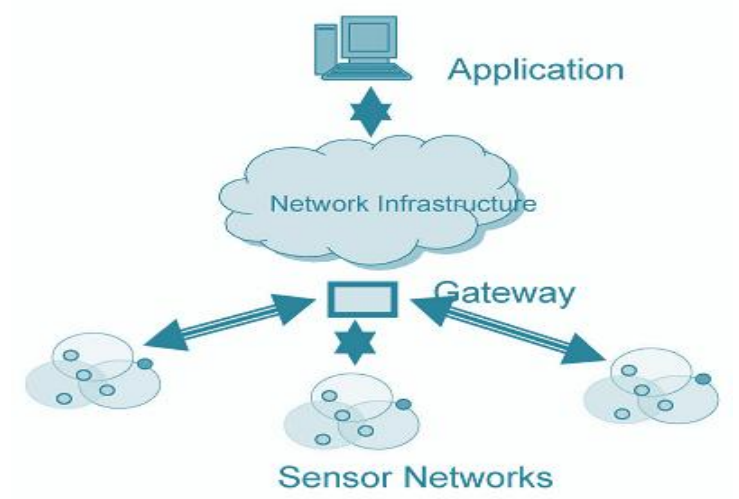

Figure 1. Basic Architecture of Wireless Sensor Network. (Ref [6]) 
Each sensor node in a sensor network is typically equipped with a radio transceiver or other wireless communications device, a processing unit which can be a small microcontroller, sensing unit, and an energy source, usually an alkaline battery. Sometimes, a mobilizer is needed to move sensor node from current position and carry out the assigned tasks. Since the sensor may be mobile, the base station may require accurate location of the node which is done by location finding system. The size of a single sensor node can vary from shoebox-sized nodes down to devices the size of grain of dust. [6]

The edificesteps of this paper is as follows. The Introductory Section ends with a brief introduction of wireless sensor network and basic principles.

In Section II, we address the characteristics and design goals for wireless sensor network.

Section IIIgives a detailed analysis of symmetrical and data centralized wireless sensor network.It mainly explain the behaviour of Directed Diffusion protocol, Sensor Protocols for Information via Negotiation and EnergyAware Data-Centric Routing.

In Section IV, we analysethe properties of rank based and hierarchical routing protocols, including LEACH, PEGASIS, HEED, DEEC, TEEN, APTEEN etc

Section V explainsthe resource heterogeneity aware routing protocols for wireless sensor network. We also explain how heterogeneity can affect the system performance.

Section VI reviews mobile and artificial intelligence based routing in WSN, the quality of service based routing is analysed in section VII and a general conclusion of the paper is in Section VIII, at the last tabular comparison for different sections are presented before references.

\section{SYMMETRICAL\&DATA CENTRALIZED ROUTING Protocols FOR WSN}

One of the most significant challenges in wide-scale Wireless Sensor Networks (WSNs) is to achieve energy efficiency in order to increase their lifetime and ensuring responsiveness. A very widely applied approach in this regard is to overload the region with numerous low-cost sensing devices that can communicate with each other wirelessly and coordinate amongst themselves in developing schedules for being asleep or active.

Directed Diffusion Protocol for wsn

Directed diffusion is a data-centric routing protocol for sensor query dissemination and processing. It is energyefficient, scalable and robust. A sensing task is described by a list of attribute- value pairs. The sink specifies a low data rate for incoming events at the beginning of the directed diffusionprocess. The sink can thereafter reinforce one particular sensor to send events with a higher data rate by resending the original interest message with a smaller interval $[14,15]$.

Sensor Protocols for Information via Negotiation

SPIN protocol was developed to overcome the problems like implosion and overlap caused by flooding protocols. The SPIN protocols are able to compute the energy consumption required to compute, send, and receive data over the network.

SPIN uses meta-data as the descriptors of the data that the sensors want to disseminate. The notion of meta-data avoids the occurrence of overlap given the sensors can name the interesting portion of the data they want to get. The size of the Meta data should be less than that of the corresponding sensor data. PIN-1(SPIN_PP) and SPIN2(or SPIN-EC) uses a resource -aware mechanism for energy savings $[17,30]$.

Energy-Aware and effective Data-Centric Routing

EAD is energy aware and helps extend network lifetime.EAD is a distributed routing protocol, which builds a virtual backbone composed of active sensors that are responsible for in-network data processing and traffic relaying.

The network is represented by a broadcast tree spanning all sensors in the network and routed at the gateway, in which all leaf nodes' radios are turned off while all other nodes correspond to active sensors forming the backbone and thus their radios are turned on[8].

\section{RANK BASED AND HIERARCHICAL ROUTING PROTOCOLS FOR WSN}

Hierarchical clustering in WSN is an energy efficient protocol with three main elements sensor nodes $(\mathrm{SN})$, base station (BS) and cluster heads $(\mathrm{CH})$ The SNs are sensors deployed in the environment to collect data. The main task of a $\mathrm{SN}$ in a sensor field is to detect events, perform quick local data processing, and transmit the data. The BS is the data processing point for the data received from the sensor nodes, and from where the data is accessed by the enduser.

Low Energy Adaptive Clustering Hierarchy (LEACH)

LEACH was the first dynamic energy efficient cluster head protocol proposed for WSN using homogeneous stationary nodes $[31,32]$.

In $\mathrm{LEACH}$ all nodes have a chance $\mathrm{CH}$ and therefore energy spent is balanced for every node. The $\mathrm{CH}$ for the Clusters are selected based on their energy load. After its election, the $\mathrm{CH}$ broadcasts a message to other nodes, which decide which cluster they want to belong to, based on the signal strength of the $\mathrm{CH}$. The clusters are formed dynamically in each round and the data collection is centralised. A TDMA schedule created by the $\mathrm{CH}$ is used to gather data from the sensors.

\section{Load-Balanced Clustering Algorithm}

In it, utilizing clustering algorithms to form a hierarchical network topology is a common method of implementing network management and data aggregation in WSNs. Assuming that the residual energy of nodes follows the random distribution, author propose a load-balanced clustering algorithm for WSNs on the basis of their distance and density distribution, making it essentially different from the previous clustering algorithms [37].

Power-Efficient Gathering in Sensor Information Systems PEGASIS is an extension of the LEACH protocol, and simulation results show that PEGASISis able to increase the lifetime of the network twice as much as the LEACH protocol.

PEGASIS forms chains from sensor nodes, each node transmits the data to neighbour or receives data from a neighbour and only one node is selected from that chain to transmit data to the BS. The data is finally aggregated and sent to the BS. PEGASIS avoids cluster formation, and assumes that all the nodes have knowledge about the 
network, particularly their positions using a greedy algorithm. [27].

Self-organizing protocol for wsn

Subramanian and Katz [38] not only describe a selforganizing protocol but develop taxonomy of sensor applications as well. Based on such taxonomy, they have proposed architectural and infrastructural components necessary for building sensor applications. The architecture supports heterogeneous sensors that can be mobile or stationary. Some sensors, which can be either stationary or mobile, probe the environment and forward the data to designated set of nodes that act as routers.

Threshold Sensitive Energy Efficient Sensor Network Protocol

TEEN is an energy efficient hierarchical clustering protocol which is suitable for time critical applications TEENhas SNs reporting data to $\mathrm{CHs}$. The $\mathrm{CH}$ sends aggregated data to the next higher level $\mathrm{CH}$ until data reaches the sink. TEEN is designed for reactive networks, where the sensor nodes react immediately to sudden changes in the value of the sensed attribute. Sensor nodes sense the environment continuously, but data transmission is done occasionally and this helps in energy efficiency [10].

Adaptive Periodic Threshold Sensitive Energy Efficient Sensor Network Protocol

APTEEN is an improvement to TEEN and aims at periodic data collection and reacting to time critical events. It is a hybrid clustering based protocol and supports different types of queries like

- $\quad$ Historical query, to get results on past data.

- One-time query that gives a snapshot of the environment and,

- $\quad$ Persistent queries, to monitor an event for a time period. The cluster head selection in APTEEN is based on the mechanism used in LEACH-C. The cluster exists for an interval called the cluster period, and then the BS regroups clusters, at the cluster change time. [9].

Hybrid, Energy-Efficient Distributed Clustering

HEED is an extension of LEACH and uses residual energy and node degree or density asymmetric for cluster selection to achieve power balancing. HEED has the following features.

- $\quad$ Prolongs network lifetime by distributing energy consumption,

Terminates clustering process within a constant number of iterations,

- $\quad$ Minimizes control overhead and

- Produces well distributed $\mathrm{CHs}$ and compact clusters.

HEED selects $\mathrm{CHs}$ based on the residual energy of the SNs and intra-cluster communication cost as a function of cluster density or node degree. HEED clustering improves network lifetime over LEACH clustering randomly selects $\mathrm{CHs}$ and cluster size and therefore nodes die faster [23, 24].

Distributed Clustering Technique for wsn

Distributed Clustering or clustered aggregation is a protocol for reactive networks and the first in-network aggregation algorithm exploiting spatial correlation, which trades a negligible quality of result (precision) for a significant energy saving. Distributed Clustering forms clusters of nodes sensing similar values [36].Updated Distributed Clustering Algorithm (updated CAG) [37] is an improvement of Distributed Clustering algorithm, where the clusters are still formed from nodes sensing similar values within a given threshold.

\section{RESOURCE HETEROGENEITY AWARE ROUTING PROTOCOLS FOR WSN}

Heterogeneous-based protocols used for heterogeneous networks where there are two types of sensors namely line-powered sensors that have no energy constraint, and battery-powered sensors having limited lifetime. The battery powered sensors have limited energy and so protocols should minimize their data communication and computation. We present some heterogeneous-based protocols in this section.

Stable Election Protocol

Author study the impact of heterogeneity of nodes, in terms of their energy, in wireless sensor networks that are hierarchically clustered. In these networks some of the nodes become cluster heads, aggregate the data of their cluster members and transmit it to the sink. Author assume that a percentage of the population of sensor nodes is equipped with additional energy resources - this is a source of heterogeneity which may result from the initial setting or as the operation of the network evolves. Authors also assume that the sensors are randomly (uniformly) distributed and are not mobile, the coordinates of the sink and the dimensions of the sensor field are known [39].

Cluster-Head Relay Routing

CHR Routing protocol uses two types of sensors to form a heterogeneous network with a single sink:

- A large number of low-end sensors denoted by $\mathrm{L}$ sensors and

- A small number of powerful high-end sensors denoted by H-sensors

Both types of sensors are static and location aware. These sensors are randomly deployed over the environment and CHR partitions the heterogeneous network into clusters or groups of sensors with L-sensors and headed by an $\mathrm{H}$ sensor [33].

Topology Control Based on Irregular Sensor Model

A deployment and topology control method is presented for heterogeneous sensor nodes with different communication and sensing range. It is based on the irregular sensor model used to approximate the behavior of sensor nodes. Besides, a cost model is proposed to evaluate the deployment cost of heterogeneous WSN. According to experiment results, the proposed method can achieve higher coverage rate and lower deployment cost for the same deployable sensor nodes [40].

Information-Driven Sensor Query based routing

IDSQ maximises information gain and minimises detection latency and energy consumption for target localization and tracking by dynamic sensor querying and data routing. In order to conserve energy only a subset of sensors are active at times when there are critical events to report in some parts of the sensed network. The choice of this active subset of sensors is balanced by the communication costs needed for communication of all sensors. A leaded is selected in this protocol that decides 
the optimal subset of sensors necessary for information sensing from the network[22, 26].

\section{MOBILITY AND INTELLIGENCE BASED ROUTING PRotocols FOR WSN}

Due to the constraints on data processing and power consumption, the use of artificial intelligence has been historically discarded in these kind of networks. However, in some special scenarios the features of neural networks are appropriate to develop complex tasks such as path discovery. Also, mobility based protocols have mobile sinks that are responsible for data collection from the network. In this section, we discuss mobility-based protocols that aim at energy efficiency.

SIR: A New Wireless Sensor Network Routing Protocol Based on Artificial Intelligence

Currently, Wireless Sensor Networks (WSNs) are formed by hundreds of low energy and low cost micro-electromechanical systems. Routing and low power consumption have become important research issues to interconnect this kind of networks. However, conventional Quality of Service routing models, are not suitable for ad hoc sensor networks, due to the dynamic nature of such systems. This research introduces a new QoS-driven routing algorithm, named SIR: Sensor Intelligence Routing.

Genetic Algorithm-Based Energy-Efficient Adaptive Clustering Protocol

The GA-based protocol is based on LEACH, called LEACH-GA herein, which basically has set-up and steady-state phases for each round in the protocol and an additional preparation phase before the beginning of the first round. In the period of preparation phase, all nodes initially perform cluster head selection process and then send their messages with statuses of being a candidate cluster head or not, node IDs, and geographical positions to the base station.

An Adaptive Data-transfer Protocol with Data Mules

This paper deal with energy-efficient data collection in sparse sensor networks with data mules. It analyse the problem of optimal data transfer from sensors to data mules, and derive an upper bound for the performance of ARQ-based data-transfer protocols. This analysis shows that protocols currently used have low performance, which results in unnecessary energy consumption.

Scalable Energy-Efficient Asynchronous Dissemination

Thisis proposed to trade-off between minimizing the forwarding delay to a mobile sink and energy savings. The source sensor reports sensed data to multiple mobile sinks and the protocol consists of three main components namely:

\section{$\bullet$}

$\bullet$

- Maintaining links to mobile sinks.

SEAD assumes that sensors are aware of their own geographic locations. Data dissemination tree is built for every sensor routed at itself and all the dissemination trees for other sensor nodes are constructed separately. SEAD sits on top of a location aware routing protocol and can be viewed as an overlay network [13].

\section{QUality OF SERVICE BASED MULTIPATH ROUTING PROTOCOLS FOR WSN}

Quality of Service (QoS) requirements like delay, reliability and fault tolerance are as important in routing in WSNs as energy efficiency. A routing protocols that support QoS with energy efficiency is discussed in this section.

Sequential Assignment Routing (SAR) protocol

One of the routing protocols that were proposed early on, which provides some QoS, is the Sequential Assignment Routing (SAR) protocol [45]. The objective of the SAR algorithm is to make the network energy-efficient and fault tolerant. SAR uses multi-hop routing and maintains routing tables to record information about its neighbors. To create multiple paths from each node to the sink, multiple trees are constructed, rooted from onehopneighbor of the sink.

SPEED QoS routing Protocol

SPEED [46] is designed to support soft real-time communication service by maintaining the desired delivery speed across the network so that the end-to-end delay is minimized. Each node keeps information only about its immediate neighbors and utilizes geographic location information to make localized routing decisions. Hence, the protocol is called "stateless," as it does not use routing tables, which result in minimal memory usage.

Energy-Aware QoS Routing Protocol

Real-time traffic is generated by imaging sensors in this QoS energy aware routing protocol [19].This protocol finds the least cost and energy efficient path and the link cost is a function that captures the nodes energy reserve, transmission energy, error rate and some communication parameters. The queuing model allows service sharing for real time and non-real-time traffic. This algorithm performs well with respect to QoS and energy metrics.

Message-Initiated Constrained-Based Routing

A Message-Initiated Constrained-Based Routing (MCBR) mechanism is proposed in [25]. MCBR is composed of explicit specifications of constraint-based destinations, route constraints and QoS requirements for messages, and a set of QoS aware meta-strategies. The separation of routing specifications and routing strategies makes it possible for exploring Meta routing strategies. This allows for there to be quality-of-service (QoS) requirements in the application layer for individual messages [47].

Energy efficient and QoS aware multipath based routing

One of the recently proposed QoS based routing protocols, specifically for wireless sensornetworks, is an energy efficient and QoS aware multipath based routing (EQSR) [48], whichprovides service differentiation by giving realtime traffic absolute preferential treatment overthe nonreal-time traffic. EQSR uses the multi-path paradigm together with a Forward ErrorCorrection (FEC) technique to recover from node failures without invoking networkwide floodingfor path-discovery.

\section{CONCLUSION\& Discussion}

Wireless Sensor Network technology offers significant potential in numerous application domains. The applications for WSNs are many and varied. They are used in commercial and industrial applications to monitor data that would be difficult or expensive to monitor using wired 
sensors. Typical applications of WSNs include monitoring, tracking, and controlling. Some of the specific applications are habitat monitoring, object tracking, nuclear reactor controlling, fire detection, traffic monitoring and so on. Given the diverse nature of these domains, it is essential that WSNs perform in a reliable and robust fashion. I believe, wireless sensor network has proved its usage in the future distributed computing environment. However, there are significant amount of technical challenges and design issues those needs to be addressed. One of the biggest challenges is the designing of efficient network management architecture to continuously support WSNs for providing services for various sensor applications.

This paper firstly proceeds to categorize routing protocols in wireless sensor networks. This taxonomy includes flat, hierarchical, QoS, and location-based routings and numerous other. After that, it discusses several multi-path routing mechanisms used to enhance network metrics such as lifetime and latency. These mechanisms are mainly divided into link-disjoint and node disjoint strategy. Using multi-path method is an effective strategy to avoid network partitioning phenomenon and prolong sensors' lifetime. An overview and comparison of various routing protocols in WSNs are also presented.

\section{REFERENCES}

[1] Yick, J.; Mukherjee, B.; Ghosal, D. Wireless Sensor Network Survey. Comput. Netw. 2008, 52, 2292-2330.

[2] Couto, D.S.J.D.; Aguayo, D.; Bicket, J.; Morris, R. A HighThroughput Path Metric for Multi-Hop Wireless Routing. Wirel. Netw. 2005, 11, 419-434.

[3] Chen, D.; Varshney, P.K. QoS Support in Wireless Sensor Networks: A Survey. In Proceedings of the International Conference on Wireless Networks, (ICWN '04), Las Vegas, NV, USA, 21-24 June 2004; pp. 227-233.

[4] Al-Karaki, J.N.; Kamal, A.E. Routing Techniques in Wireless Sensor Networks: A Survey. IEEE Wirel. Commun. 2004, 11, 6-28.

[5] Akkaya, K.; Younis, M. A Survey on Routing Protocols for Wireless Sensor Networks. Ad Hoc Netw. J. 2005, 3, 325-349.

[6] I. Akyildiz, W. Su, Y. Sankarasubramaniam, and E. Cayirci, "A survey on Sensor Networks," IEEE Communications Magazine, vol. 40, Issue: 8, pp. 102-114, August 2002. Available from WWW: http://citeseer.ist.psu.edu/akyildiz02survey.html

[7] $\mathrm{Yu}$ Mengjie, Mokhtar H., Merabti M., "A Survey of Network Management Architecture in Wireless Sensor Network"

[8] A. Boukerche, X. Cheng, and J. Linus, "Energyaware data - centric routing in micro-sensor networks", Proceedings ACM MSWiM, in conjunction with ACM MobiCom, San Diego, CA, Sept. 2003

[9] A.Manjeshwar and D.P. Agarwal," APTEEN: A Hybrid Protocol for Efficient Routing and Comprehensive Information Retrieval in Wireless in Wireless Sensor Networks ",in the Proceedings of the 2nd International Workshop of Parallel and Distributed Computing Issues in Wireless Networks and Mobile Computing, San Francisco CA, April 2001.

[10] A.Manjeshwar and D.P. Agarwal, "TEEN : A Protocol for Enhanced Efficiency in Wireless Sensor Networks", in the Proceedings of the 1 st International Workshop on Parallel and Distributed Computing Issues in Wireless Networks and Mobile Computing, San Francisco, CA, April 2001

[11] B. Chen, K. Jamieson, H. Balakrishnan, and R. Morris, "Span: An energy-efficient coordination algorithm for topology maintenance in ad hoc wireless networks", Proceedings ACM MobiCom'01, Rome, Italy, July 2001.

[12] B. Chen, K. Jamieson, H. Balakrishnan, and R. Morris, "Span: An energy-efficient coordination algorithm for topology maintenance in ad hoc wireless networks", Proceedings ACM MobiCom'01, Rome, Italy, July 2001.
[13] B. Karp and H.T. Kung, "GPSR: Greedy perimeter stateless routing for wireless networks', proceedings ACM MobiCom '00, Boston, MA, Aug .2000, pp. 243-254.

14] C. Intanagonwiwat, R. Govindan, D. Estrin, J.Heidemann, and F. Silva,"Directed diffusion for wireless sensor networking",IEEE/ACM Transactions on Networking, vol. 11.,no. 1, Feb. 2003.

[15] C.Intanagonwiwat, R.Govindan, and D.Estrin,"Directed diffusion: A scalable and robust communication paradigm for sensor networks", Proceedings ACM MobiCom'00, Boston, MA, Aug.2000.

[16] I.F. Akyildiz, W .Su, Y. Sankarasubramaniam, and E. Cayirci, "Wireless sensor networks: a survey", computer networks (Elsevier) Journal, Vol.38, no.4, Mar.2002, pp. 393-422.

[17] J. Kulik, W. Heinzelman, and H. Balakrishnan, "Negotiation based protocols for disseminating information in wireless sensor networks", Wireless Networks, vol. 8, no. 2/3, Mar.-May 2002.

[18] Jamal Al-Karaki, and Ahmed E.Kamal, "Routing Technique in Wireless Sensor Networks: A Survey", IEEE Communications Magazine, vol 11, no.6, Dec.2004, pp 6-28.

[19] K.Akkaya and M. Younis, "An Energy-Aware QoS Routing Protocol for Wireless Sensor Networks," in the Proceedings of the IEEE Workshop on Mobile and Wireless Networks(MNV2003),Providence, Rhode Island ,May2003.

20] Kemal Akkaya and Mohamed Younis, "A Survey on Routing Protocols for Wireless Sensor Networks", Ad hoc Networks, vol. 3, no. 3, May 2005.

[21] L. Li and J. Y. Halpern, "Minimum-energy mobile wireless networks revisited", Proceedings IEEE ICC'01, Helsinki, Finland, June 2001.

[22] M. Chu, H. Haussecker, and F. Zhao, "Scalable information-driven sensor querying and routing for ad hoc heterogeneous sensor networks", International Journal of High performance computing Applications, vol.16, no.3, Feb.2002 , pp. 293-313

[23] OssamaYounis and Sonia Fahmy "Heed: A Hybrid, energy, Distributed Clustering Approach for Ad-hoc Networks:, IEEE Transactions on Mobile Computing Issues in Wireless Networks and Mobile Computing, vol 3,no. 4, Dec 2004.pp.366-369.

[24] OssamaYounis and Sonia Fahmy, "Distributed Clustering in Adhoc sensor Networks: A Hybrid, Energy - efficient Approach", September 2002. International Journal of Computer Science.

[25] R.C.Shah, S. Roy, S.Jain, and W .Brunette, "Data MULEs: Modelling a three -tier architecture for sparse sensor networks", proceedings SN P A'03, Anchorage, AK, May 2003, pp. 30-41.

[26] S. Lindsey, C.S. Raghavendra, and K.M. Sivalingam, "Data gathering in algorithms in sensor networks using energy metrics ", IEEE Transactions on Parallel and Distributed Systems, vol. 13, no. 9,Sept. 2002, pp.924-95.

[27] S. Lindsey and C.S. Raghavendra, "PEGASIS Power-efficient Gathering in Sensor Information System", Proceedings IEEE Aerospace Conference, vol. 3, Big Sky, MT, Mar. 2002, pp. 1125-1130.

[28] S.K.Singh, M.P.Singh and D.K.Singh, "Energy- efficient Homogeneous Clustering Algorithm for Wireless Sensor Network", International Journal of Wireless \& Mobile Networks (IJWMN), Aug.2010, vol.2.no 3, pp 49-61.

[29] Rodoplu and T. H. Meng, "Minimum energy mobile wireless networks", IEEE Journal on Selected Areas in Communications, vol. 17, no. 8, Aug. 1999.

[30] W. R. Heinzelman, J. Kulik, and H. Balakrishnan, "Adaptive protocols for information dissemination in wireless sensor networks", Proceedings ACM MobiCom '99, Seattle, WA, Aug.1999.

[31] W.R. Heinzelman, A. Chandrakasan, and H. Balakrishnan, "Energy- efficient Communication Protocol for Wireless Microsensor Networks", in IEEE Computer Society Proceedings of the Thirty Third Hawaii International Conference on System Sciences (HICSS '00), Washington, DC, USA, Jan. 2000, vol. 8, pp. 8020

[32] W.R. Heinzelman, A. Chandrakasan, and H. Balakrishnan, "An Application-Specific Protocol Architecture for Wireless Microsensor Networks" in IEEE Tmnsactions on Wireless Communications (October 2002), vol. 1(4)

[33] X. Du and F. Lin, "Improving routing in sensor networks with heterogeneous sensor nodes", Proceedings IEEE VTC '05, Dallas TX, Sept 2005

[34] Y.Xtu, J. Heidemann and D.Estrin, "Geographyinformed energy conservation for ad-hoc routing", Proceedings ACM/IEEE MobiCom '01, Rome, Italy, July 2001.

[35] Y.Yu, R.Govindan and D.Estrin, "Geographical and energy aware routing: A recursive data dissemination protocol for wireless sensor 
networks: Technical Report, UCLA/CSD-TR-01- 0023, UCLA, Computer Science Department, Mat 2001.

[36] Yoon S., Shahabi C., "Exploiting Spatial Correlation towards an Energy Efficient Clustered Aggregation Technique (CAG)", IEEE Conference on Communications, 2005.

[37] Ying Liao, Huan Qi, and Weiqun L, "Load-Balanced Clustering Algorithm withDistributed Self-Organization for Wireless Sensor Networks", IEEE SENSORS JOURNAL, VOL. 13, NO. 5, MAY 2013.

[38] L. Subramanian, R.H. Katz, "An architecture for buildingselfconfigurablesystems", IEEE/ACM Workshop on Mobile Ad Hoc Networking and Computing, Boston, MA, August 2000.

[39] GeorgiosSmaragdakis Ibrahim MattaAzerBestavros, "SEP: A Stable Election Protocol for clustered heterogeneous wireless sensor networks", Technical Report BUCS-TR-2004-022.

[40] Chun-Hsien Wu, "Heterogeneous Wireless Sensor Network Deployment and Topology Control Based on Irregular Sensor Model", Department of Computer Science, National Tsing Hua University.

[41] Kumar, P., "Geographical location based hierarchical routing strategy for wireless sensor networks", Devices, Circuits and Systems (ICDCS), 2012 International Conference.

[42] RafeAlasem, Ahmed Reda, Mahmud Mansour, "Location based energy-efficient reliable routing protocol for wireless sensor networks", Recent Researches in Communications, Automation, Signal Processing, Nanotechnology, Astronomy and Nuclear Physics.

[43] Julio Barbancho, Carlos León, Javier Molina, Antonio Barbancho, "SIR: A New Wireless Sensor Network Routing Protocol Based on Artificial Intelligence", Advanced Web and Network Technologies, and ApplicationsLecture Notes in Computer Science Volume 3842, 2006, pp 271-275.

[44] Jenn-Long Liu and Chinya V. Ravishankar, "LEACH-GA Genetic Algorithm-BasedEnergy-Efficient Adaptive Clustering Protocol for wireless sensor network"

[45] K. Sohrabi, J. Pottie, Protocols for self-organization of a wireless sensor network, IEEE Personal Communications 7 (5) (2000) 16-27.

[46] T. He, et al., SPEED: A stateless protocol for real-time communication in sensor networks, in: The Proceedings of the International Conference on Distributed Computing Systems, Providence, RI, USA, May, 19-22, 2003, pp.46-55.

[47] Y. Zhang, M. Fromherz, Message-initiated constraint-based routing for wireless adhoc sensor networks, in: The Proceedings of the First IEEE Consumer Communication and Networking Conference, CCNC-2004, LasVegas, Nevada, USA, January, 5-8, 2004, pp.648-650.

[48] alel Ben-Othman, Bashir Yahya "Energy efficient and QoS based routing protocol for wireless sensor Networks", J.Parallel Distributed computing (70), P-849-857, March, 2010.

\section{BIOGRAPHIES}

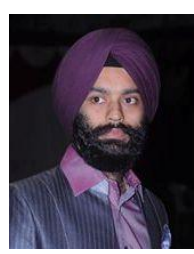

Mr Gurpreet Singh Chhabra having Master degree in Computer Science and is a member of CSI (Computer Society of India) and is pursuing $\mathrm{Ph} \mathrm{D}$ in Wireless Sensor network(WSN). He has a credit of many national and international papers. His qualifications are fortified with a great deal of creativity and problem solving skills. He has also a proven record of teaching for the last 7 years with presently working as Head of Department (CSE).

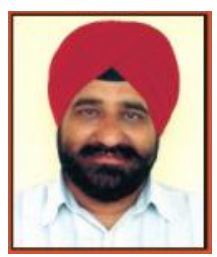

Dr Pushpinder Singh Patheja after completing his Ph D from Maulana Azad National Institute of Technology, Bhopal. He has also a proven record of teaching software technology for the last 22 years with presently working as Dean (M Tech Courses) at BIST, Bhopal. He has delivered "Expert Lecturer" at NIT, Bhopal \& Calicut. He has been appointed as a reviewer for CSNT-2011, a conference in which papers were published in IEEE Journal held at Jammu and was also in Program Committee for WICT 2011 (World Congress on Information and Communication Technologies) and WICT 2013,14 organized by MIRLABS journals and conferences. He has presented more than 35 papers at both National and International level. 